\title{
Analysis of Data Collected from Software Development Case Studies
}

\author{
Robin Hunter \\ Department of Computer Science, University of Strathclyde, Richmond Street, Glasgow,UK.
}

\begin{abstract}
The ESPRIT project, SCOPE, on software product evaluation, collected data from 27 case studies in order to experiment with and evaluate software assessment modules. Data from 14 of the case studies was transferred to a central database held at the University of Strathclyde. Data in the database belonging to individual case studies was analysed in a number of ways within the SCOPE project, and a limited amount of analysis across the case studies was also performed. Since the project was completed, further analysis has been performed across the case studies with the aid of the database's SQL interface. The paper describes some of the results obtained and points to how further useful analysis might be performed.
\end{abstract}

Keyword Codes: D.2.8

Keywords: Metrics

\section{INTRODUCTION}

The SCOPE (Software CertificatiOn Project for Europe) was an ESPRIT project funded by the European Community from 1989 until 1993. The Project was led by Verilog, a software tool developer based in Toulouse, France. There were eleven other partners and four associate partners involved in the project from a total of eight European countries. One of the partners was the University of Strathclyde in Glasgow. The Computer Science Department in the University was involved in the project from a Software Engineering point of view, while the Law School in the University was involved in legal aspects of the project. The SCOPE database, which will be discussed in this paper, was also developed (in conjunction with other partners) at Strathclyde.

The SCOPE project was concerned with software product evaluation for certification. Thus it contrasted with (and complemented) other concurrent projects such as the UK based TickIT project based on the application of ISO 9001[7] to the software process, and the work of the SPICE (Software Process Improvement and Capability dEtermination) Project based on the work of the US Software Engineering Institute and its Capability Maturity Model[5].

The approach taken by the SCOPE project was based on the Software Product Standard ISO 9126[8] which identified six quality characteristics for software products viz Portability, Efficiency, Reliability, Functionality, Usability and Maintainability. Prior to SCOPE, the main initiatives concerning software product quality were compiler validation which was based on language standards, and the German Gutegemeinschaft Software scheme based on the German Standard DIN 66 285(now ISO 12119)[9]. This scheme provided a basic assessment of the functionality of software packages mainly to ensure that the package description (required by the Standard) accurately described the behaviour of the software.

SCOPE was more ambitious than either of these schemes in that it was concerned with quality characteristics other than functionality. It was also modular in its approach, in that it defined stand alone modules that were able to assess a particular quality characteristic, to a particular level, by a particular method. 


\section{ASSESSMENT MODULES}

The SCOPE assessment modules were mostly of one of three forms: modules based on static analysis, modules based on dynamic analysis, modules based on predefined check-lists.

\subsection{Modules based on Static Analysis}

These modules used results obtained by measuring source code statically (ie without running it). Two static analysers were used, Logiscope[12] and QUALMS[13], Logiscope evaluated a range of metrics from Halstead and McCabes' metrics to call graph metrics and certain types of user-defined metrics. QUALMS was mainly concerned with control graph metrics. Data from the static analysers was used in connection with the derivation of evaluation modules for quality characteristics such as maintainability and functionality.

\subsection{Modules based on Dynamic Analysis}

Two types of modules were developed based on dynamic analysis. One type was for reliability modelling and the other dealt with test coverage. Our understanding of reliability differed somewhat from that of ISO 9126 . We used the generic definition of reliability being the probability that a software system would not fail in a given period under a given usage. Using this definition reliability may be predicted with the aid of a reliability growth model of which there are a number available. The project made use of a commercially available product (details in acknowledgement at end of paper) which chooses, on the basis of best fit so far, which of nine models should be used to predict the future reliability of a system.

Test coverage was chosen as a measure of functionality and a number of code coverage measures were defined. It was realised that, this type of data would be most easily collected by means of a dynamic analysis tool with capabilities such as Logiscope provides, integrated with its static analysis capabilities.

\subsection{Modules based on check-lists}

Many of the evaluation modules were based on check-lists supported by a check-list manager tool used by the project. Initially most of the check-lists were developed by a single partner for a single case study. However towards the end of the project there was a merging of ideas and a number of check-lists were produced which represented the consensus view of the project as a whole, being in many cases, amalgams of existing checklists.

\section{THE CASE STUDIES}

The SCOPE evaluation modules were experimented with, validated and refined through 27 software development case studies monitored in eight European countries. The data collected from the case studies was analysed locally and/or centrally at the University of Strathclyde.

\section{DATA COLLECTION AND STORAGE}

\subsection{Data collection}

SCOPE was fortunate to have two phases, the first one of 18 months (March 89 - September 90 ) and the second one of 34 months (October 90 - June 93). Six of the case studies were in phase 1 thus the project had the opportunity to experiment with data collection in phase 1 and to try and 'get it right' in phase 2.

A number of lessons were learnt from phase 1[10]. different tools can give different values for the same measurement naming software product parts is not trivial data required is often held by tools, making manual collection redundant 


\subsection{Data transfer}

In many cases data had to be transferred from a software development or analysis tool to a central repository. In practice a stream of metrics were transferred at a time and a simple interface language was defined to represent such a stream.

\subsection{Data storage}

In phase 1 of SCOPE the repository used for the data held centrally was a relational database with an object-oriented interface developed by the REQUEST Project[3]. This was successful, but in phase 2 the use of a simple relational data base was attractive in that it would be more portable, would offer a more usable SQL interface for exploratory investigations, and would be more likely to be compatible with other software data collection initiatives.

Along with the database were developed, with the aid of the Unix compiler tools, simple routines for transferring metric data streams to and from the database.The metrics mainly comprised static analysis metrics, code coverage metrics and reliability data

\section{DATA ANALYSIS}

\subsection{Analysis performed for individual case studies}

It is not possible to describe all the analysis performed by SCOPE partners and their case study providers on individual case studies. One case study used the inter-failure times of the most serious failures from failure data describing 1010 failures, to identify the 'best fit' reliability growth model using the reliability module already described. The model identified in this case was the Goel-Okumoto model[2]. Other investigations involved seeking correlations between metrics collected for a particular case study. Test coverage data for one case study, for example, was found to correlate with McCabe's metric[11], depth of nesting and largest prime metrics.

\subsection{Analysis performed centrally}

Certain basic checks were performed on case study data when it as transferred to the central site. These were mainly to ensure consistency of data and to catch any obvious 'problems'. In addition, static metrics were analysed to detect anomalies, as well as for indicators of structuredness, maintainability etc.

\subsection{Analysis performed across case studies during SCOPE}

A number of simple comparisons across the case studies were made centrally. Five case studies provided both Logiscope and Qualms metrics, three provided only Logiscope metrics and five case studies only Qualms metrics. Bar graphs and histograms showing characteristics of the various case studies such as mean and maximum values of numbers of statements per module, depth of nesting, values of various complexity metrics were produced for all the case studies for which data was available. These were produced mainly to identify case studies with anomalous values of these metrics compared with the case studies as a whole.

\subsection{Analysis performed after the end of SCOPE}

Just before the end of SCOPE the database was 'sanitised' in order that none of the data in the data base, or results from it which might later be published, could be traced to the original supplier of the data. In particular, program names, identifier names etc. were all replaced by symbols of no mnemonic significance. Some exploratory analysis of the data has been performed at the University of Strathclyde and in this section we describe the work done so far. It falls into a number of categories.

visual browsing: Visual browsing was used to confirm the reasonableness of the data, for example that the total number of operands was never less than the number of distinct operands. It was also used to look for possible correlations deserving further investigation. 
effect of language on metric values: Some simple analysis was performed to look for correlations between particular metric values and the source language in which the software was written. No such correlations were found.

Halstead's metrics : Halstead's length metric is known [1] not to correlate well with the actual length of a source code program or module. Analysis of the Case Studies confirmed this lack of correlation.

browsing with $S Q L$ : SQL proved to be a powerful tool in detecting anomalies and possible correlations in the database. For example it was easy to show that our two static analysers did not always produce consistent results as has been shown by Hunter and Roper[6], where it was concluded that the inconsistencies stemmed from inadequate definitions of the metrics, rather than from deficencies in the tools themselves.

\section{CONCLUSIONS}

We have tried to identify some of the issues involved in implementing a simple metrics database. We feel that some of the issues are not generally appreciated and it is not surprising that few such databases currently exist. SCOPE was successful in setting up a database containing a large number of source code metrics by paying close attention to the issues of data collection, data transfer and data analysis described earlier. In particular the use of SQL as a browsing tool is advocated in situations where it is not known in advance what types of analysis will be performed. A database of the type described might be used industrially to predict size, cost and other attributes of a software product to be developed, based on comparisons of known attributes of previously developed software products and the new product to be produced. It is from exploratory analysis of the type described in this paper that useful predictors, perhaps specific to a particular environment, may be found.

\section{Acknowledgements}

The contributions of the following towards the data capture, transfer and storage work of the SCOPE project at the University of Strathclyde is gratefully acknowledged: Paul Carson, Stephen Church, Kenneth Kirkwood, Martin Neil, Andrew Rae and Marc Roper.The support of Reliability and Statistical Consultants Ltd, Surrey, UK in supplying their reliability programs under special terms is also gratefully acknowledged.

\section{REFERENCES}

1. Bache, R. and Bazzana, G.: 'Software Metrics for Software Assessment', (McGraw Hill, 1994)

2. Goel, A. L. and Okumoto, K.: "Time-dependent error-detection rate model for software reliability and other performance measures', IEEE Trans. Reliability, 1979

3. Dale, C.: "The REQUEST Database for Software Reliability and Software Development Data', 4th annual ESPRIT conference, Brussels, 1987

4. Halstead, M.: 'Elements of Software Science', (Elsevier North Holland, 1977)

5. Humphrey, W. S.: 'Managing the Software Process', (Addison Wesley, 1989)

6. Hunter, R. B. and Roper, R. M. F.: 'Standardisation of Source Code Metrics', EUROMETRICS91, Paris, 1991

7. ISO 9001, 'Quality Systems, Model for Quality Assurance in Design/Development, Installation and Servicing', (International Standards Organisation, 1987)

8. ISO 9126, 'Software Product Evaluation - Quality Characteristics and Guide-lines for their Use', (International Standards Organisation, 1987)

9. ISO 12119, 'Information Technology-Software Packages - Quality requirements and testing', (International Standards Organisation, 1994)

10. Kirkwood, K. B., Hunter, R. B. and Rae, A. K.: 'Experiences of Automated Data Collection from Software Development Projects', Proceedings of the IFIP WG 5.4 Conference on Approving Software Products(ASP 90), Garmisch-Partenkirchen, published by North Holland, 1990

11. McCabe, T. J.: 'A Complexity Measure', IEEE Trans. in Software Engineering, vol 2, 1976

12. Verilog; Logiscope Technical Presentation, Toulouse, 1989

13. Wilson. L. and Leelasena. L.: The QUALMS program documentation, Alvey project SE/69, South Bank Polytechnic, London, 1988 